\title{
Unbalanced MMP/TIMP-1 expression during the development of experimental pulmonary fibrosis with acute paraquat poisoning
}

\author{
BO-LIANG WANG ${ }^{1}$, YAN-YANG TU ${ }^{1}$, JIAN-FANG FU ${ }^{4}$, YUE-XIA ZHONG ${ }^{1}$, \\ GUO-QIANG FU ${ }^{1}$, XIAO-XI TIAN ${ }^{1}$, LI-HE WANG ${ }^{1}$, LI GONG $^{2}$ and QIN-YOU REN ${ }^{3}$ \\ Departments of ${ }^{1}$ Emergency; ${ }^{2}$ Pathology; ${ }^{3}$ Traditional Chinese Medicine, Tangdu Hospital, \\ the Fourth Military Medical University, Shaanxi 710038; ${ }^{4}$ Department of Endocrinology, \\ Xijing Hospital, the Fourth Military Medical University, Shaanxi 710032, P.R. China
}

Received September 24, 2010; Accepted January 6, 2011

DOI: $10.3892 / \mathrm{mmr} .2011 .425$

\begin{abstract}
Paraquat (PQ)-induced pulmonary toxicity is known to result in pulmonary edema, infiltration of inflammatory cells and damage to the alveolar epithelium, which may progress to severe fibrosis. Matrix metalloproteinases (MMPs) and their physiological inhibitors, tissue inhibitors of matrix metalloproteinases (TIMPs), which degrade and remodel the excess extracellular matrix, are believed to play an important role in the development of fibrotic tissue. In this study, we examined the sequential expression of MMP-2, MMP-9 and TIMP-1 in a rat model of pulmonary fibrosis induced by PQ. Adult male Sprague-Dawley rats were treated intraperitoneally with PQ $(20 \mathrm{mg} / \mathrm{kg}$ ) and saline (control group). Rats were sacrificed at days 1, 3, 7 and 21 after the PQ treatment. Lungs were excised for histological evaluation and immunohistochemical analyses, as well as the determination of collagen content, gene expression by fluorimeter-based quantitive RT-PCR assay and gelatinolytic activity by zymography. Lung MMP-2 and -9 mRNA expression progressively increased and reached a peak on day 7 after PQ treatment, while TIMP-1 mRNA levels in the PQ-treated lungs reached a peak on day 21 after modeling. Lung zymography revealed an increase in progelatinase $\mathrm{B}$, progelatinase $\mathrm{A}$ and their active forms. In conclusion, unbalanced MMP/TIMP-1 expression and excessive gelatinolytic activity contribute to PQ-induced pulmonary fibrosis. Their precise role should be studied in depth as they may represent relevant therapeutic targets for $\mathrm{PQ}$ poisoning-induced pulmonary fibrosis.
\end{abstract}

Correspondence to: Professor Bo-Liang Wang, Department of Emergency, Tangdu Hospital, the Fourth Military Medical University, Xi'an, Shaanxi 710038, P.R. China

E-mail: boliang_wang@yahoo.cn

Professor Qin-You Ren, Department of Traditional Chinese Medicine, Tangdu Hospital, the Fourth Military Medical University, Xi'an, Shaanxi 710038, P.R. China

E-mail: renqinyou@fmmu.edu.cn

Key words: paraquat, matrix metalloproteinase, tissue inhibitors of matrix metalloproteinase, pulmonary fibrosis

\section{Introduction}

Paraquat (PQ), 1,1'-dimethyl-4'-bipyridinium ion, is a fastacting, non-selective contact herbicide absorbed by foliage with some translocation in the xylem. It is used for the broad spectrum control of broad-leaved weeds and grasses in fruit orchards and plantations, and for inter-row weed control in many crops (1). However, toxic effects of PQ have been described. Clark et al reported similar histological effects of PQ in rats, mice and dogs (2) in which the lung, liver, kidney and thymus were affected, with the lung being the major target. In forensic practice, fatal cases of acute $P Q$ poisoning are often encountered (3). PQ-induced pulmonary toxicity is known to result in pulmonary edema, infiltration of inflammatory cells and damage to the alveolar epithelium, which may progress to severe fibrosis. Pulmonary fibrosis is a chronic disease characterized by the loss of lung epithelial cells, which are replaced by interstitial myofibroblasts and the deposition of extracellular matrix proteins in the lung interstitium, leading to pulmonary structural remodeling. Even in cases in which rescue from the fatality of acute PQ poisoning is successful, chronic lung injury, such as fibrosis, occurs with a high rate of morbidity. Therefore, it is important to understand the pathogenic mechanism of PQ-induced pulmonary fibrosis.

Matrix metalloproteinases (MMPs) are proteolytic enzymes belonging to the metzincin superfamily of metalloproteinases (4), and are believed to be implicated in many physiological and pathological processes, including embryonic development, morphogenesis, reproductive processes, bone remodeling, wound healing, cancer, arthritis and atherosclerosis (5). MMP synthesis and function are regulated by three major mechanisms, including transcriptional activation, post-transcriptional processing and control of activity by a family of endogenous inhibitors, collectively known as tissue inhibitors of metalloproteinases (TIMPs) (6). In healthy lungs, MMPs and TIMPs are produced in the respiratory tract by a panel of different structural cells. Deregulation of the proteolytic-antiproteolytic network and inappropriate secretion of various MMPs by stimulated structural or inflammatory cells is believed to take part in the pathophysiology of numerous lung diseases, including asthma, chronic obstructive pulmonary disease (COPD), pulmonary fibrosis and lung cancer (7). 
MMP-9 was the first MMP to be studied in depth in relation to its implication in the pathology of asthma (8). Increased levels of MMP-9 were indeed detected in bronchoalveolar lavage fluid, sputum induced by the inhalation of hypertonic saline and the serum of asthmatic patients. Based on observations of increased levels of MMPs in asthmatic patients and taking into account the potential risk of side effects of broad spectrum inhibitors of metalloproteinases, which have led to the discontinuation of most clinical trials in the field of cancer, numerous researchers are investigating the specific roles played by individual MMPs (9).

Compared with healthy subjects, patients with COPD have a marked increase in the expression and activity of MMP-2, MMP-9 and MT1-MMP in the lung parenchyma, and increased gelatinolytic activity linked to MMP-2 and MMP-9 in the sputum (10). In bleomycin-induced pulmonary fibrosis, a temporal difference is observed in the expression and localization of MMPs and TIMPs (11). In the early stages of this disease, the gelatinolytic activity of MMP-9 appears to be predominant, and likely contributes to the disruption of the alveolar epithelial basement membrane and enhancement of fibroblast invasion to alveolar spaces. In the late stages of the disease, MMP-2 appears to become predominant. TIMP-1 gene expression is also up-regulated during fibrosis.

Although MMPs and TIMPs have been reported to play an important role in the development of fibrotic tissue, their relationship with PQ-induced pulmonary fibrosis has not yet been confirmed. Therefore, the aim of this study was to evaluate the sequential expression of MMP-2, MMP-9 and TIMP-1 in an established rat model of PQ-poisoned lungs.

\section{Materials and methods}

Animals. Animal experiments were performed according to international guidelines. The study was approved by the Institutional Animal Care and Use Committee of the Fourth Military Medical University.

Eighty adult male Sprague-Dawley (SD) rats (230-250 g) were obtained from the Experimental Animal Center of the Fourth Military Medical University, and were housed five per cage with free access to food and water in a constant environment $\left(22 \pm 2^{\circ} \mathrm{C}, 50 \pm 5 \%\right.$ humidity, $12 \mathrm{~h}$ light/dark cycle). The rats were divided into two groups of forty: the paraquat treatment group and the control group.

Chemicals and reagents. Chemicals were of analytical reagent grade. Before the experiment, all the vessels and tips for pipetting were dipped in strong $\mathrm{HNO}_{3}$ for $24 \mathrm{~h}$, and then washed with ultrapure water. The water was purified in a Milli-Q water purification system (Millipore, Bedford, MA, USA).

Modeling and tissue sampling. The rats were treated intraperitoneally with PQ (20 mg/kg; Sigma Chemicals, St. Louis, MO, USA) or with saline (control group). Animal body weights were recorded every week and visual examination of animal health was performed daily. On days 1, 3, 7 and 21 after PQ treatment, the rats were anesthetized by intraperitoneal pentobarbital $(50 \mathrm{mg} / \mathrm{kg})$. The left lungs were used for histological evaluation and immunohistochemical staining, whereas the right lungs were used for the determination of MMP-2, MMP-9 and TIMP-1 gene expression and lung tissue zymography. Ten animals from each group were studied at each time interval.

Histological evaluation. For histology and immunohistochemistry, the left lungs were instilled with $4 \%$ paraformaldehyde in PBS through a tracheal cannula at a constant pressure of $7 \mathrm{~cm}$ $\mathrm{H}_{2} \mathrm{O}$, and were then embedded in paraffin. Subsequently, the lobes of the lung were separated and sectioned sagittally into $5-\mu \mathrm{m}$-thick sections, then stained with hematoxylin and eosin (H\&E). The sections were examined by light microscopy and assessed for the presence of hemorrhage, intra-alveolar edema and fibrosis.

Hydroxyproline assay of lung tissue. The hydroxyproline contents of lung tissues were determined and the data were expressed as micrograms of hydroxyproline per milligram of lung tissue. Total lung tissues from the control and PQ-treated rats were dried and hydrolyzed in $1 \mathrm{ml}$ of $12 \mathrm{~N} \mathrm{HCl}$ for $24 \mathrm{~h}$ at $110^{\circ} \mathrm{C}$, and hydroxyproline colorimetric analysis was performed as described by Woessner (12).

Immunohistochemical staining. For immunohistochemical analysis, deparaffinized and dehydrated sections were immersed in $0.3 \% \mathrm{H}_{2} \mathrm{O}_{2}$ in methanol for $30 \mathrm{~min}$ to eliminate endogenous peroxidase activity. The sections were further incubated with PBS containing $1 \%$ rabbit normal serum and $1 \%$ bovine serum albumin to reduce non-specific reactions. The sections were then incubated with anti-F4/80 for murine macrophages or anti-CD3 for T-cells at a concentration of $1 \mathrm{mg} / \mathrm{ml}$ at $4^{\circ} \mathrm{C}$ overnight. Tissue sections were rinsed and subsequently incubated with biotinylated rabbit anti-rat $\operatorname{IgG}$. Immune complexes were visualized using the Catalyzed Signal Amplification System (Dako, Japan) according to the manufacturer's instructions.

Fluorimeter-based quantitive RT-PCR assay. The mRNA expression levels of MMP-2, MMP-9 and TIMP-1 in the lung tissues of the rats in each group were detected by fluoresceinbased quantitive RT-PCR.

Total RNA was extracted from the tissues with a phenol/ guanidine isothiocyanate-based reagent (TRIzol $^{\mathrm{TM}}$; Gibco BRL, Scotland, UK). Briefly, $1 \mathrm{ml}$ TRIzol and $200 \mu \mathrm{l}$ chloroform were added to each tube. The RNA was then precipitated with $500 \mu \mathrm{l}$ isopropanol (v/v), washed with $75 \%$ ethanol and air-dried. The purified RNA was then dissolved in $10 \mu \mathrm{l}$ diethyl pyrocarbonate (DEPC)-treated water, and the optical density was measured at a wavelength of $260 / 280 \mathrm{~nm}$ and stored at $-80^{\circ} \mathrm{C}$ prior to use in the synthesis of cDNA.

RNA was reverse transcribed using the Superscript ${ }^{\mathrm{TM}}$ Preamplification System (Life Technology, Guangzhou, China). Total RNA was mixed with oligo(dt) primers and incubated at $70^{\circ} \mathrm{C}$ for $10 \mathrm{~min}$. The mixture was then chilled on ice and incubated with a $1 \mathrm{X}$ reverse transcriptase buffer (50 mM Tris- $\mathrm{HCl}, \mathrm{pH} 8.4,75 \mathrm{mM} \mathrm{KCl}$ ), $3 \mathrm{mM} \mathrm{MgCl}, 500 \mu \mathrm{M}$ of each deoxynucleotide, $10 \mathrm{mM}$ dithiothreitol and 200 units Superscript II RT reverse transcriptase at $42^{\circ} \mathrm{C}$ for $50 \mathrm{~min}$. The 20- $\mu 1$ reactions were further incubated at $70^{\circ} \mathrm{C}$ for $15 \mathrm{~min}$ and 2 units RNase $\mathrm{H}$ was added to each tube. Following a 
Table I. Body weight (mean \pm SD) of rats in different groups

\begin{tabular}{|c|c|c|c|c|c|}
\hline & Day 0 & Day 1 & Day 3 & Day 7 & Day 21 \\
\hline Control & $201.6 \pm 10.2$ & $209.8 \pm 11.5$ & $220.4 \pm 10.8$ & $232.1 \pm 9.60$ & $241.7 \pm 10.3$ \\
\hline PQ treatment & $202.9 \pm 9.50$ & $195.6 \pm 11.3$ & $183.7 \pm 10.4$ & $176.5 \pm 11.2$ & $161.8 \pm 10.1$ \\
\hline
\end{tabular}

final incubation at $37^{\circ} \mathrm{C}$ for $20 \mathrm{~min}$, the cDNA was stored at $-80^{\circ} \mathrm{C}$ until use.

PCR was performed in the MJ Opticon Monitor 2.0 (MJ Ltd., Alameda, CA, USA) using SYBR-Green I (Biogene, Valencia, CA, USA) as the fluorescein. The following pairs of primers were used: $\beta$-actin (258 bp): 5' GAC CTT CAA CAC CCC AGC CA 3' (sense), 5' GTC ACG CAC GAT TTC CCT CTC 3' (antisense); MMP-2 (309 bp): 5' CTA TTC TGT CAG CAC TTT GG 3' (sense), 5' CAG ACT TTG GTT CTC CAA CTT 3' (antisense); MMP-9 (309 bp): 5' AAA TGT GGG TGT ACA CAG GC 3' (sense), 5' TCC TTG GGG CTC TCA ATT TC 3 ' (antisense); TIMP-1 (333 bp): 5' GAC CAC CTT ATA CCA GCG TT 3' (sense), 5' GTC ACT CTC CAG TTT GCA AG 3' (antisense). Primers were synthesized by Sangon Co. (Shanghai, China). Reverse transcription and amplification were performed using a thermocycler (Biometra, Göttingen, Germany) programmed with the following parameters: reverse transcription at $50^{\circ} \mathrm{C}$ for $30 \mathrm{~min}, \mathrm{PCR}$ activation at $95^{\circ} \mathrm{C}$ for $7 \mathrm{~min}, 40$ cycles of denaturation at $95^{\circ} \mathrm{C}$ for $30 \mathrm{sec}$, annealing at $58^{\circ} \mathrm{C}$ for $30 \mathrm{sec}$, extension at $72^{\circ} \mathrm{C}$ for $30 \mathrm{sec}$, and a final extension at $72^{\circ} \mathrm{C}$ for $10 \mathrm{~min}$.

Sample quantification was carried out by Sequence Detection System software (SDS 2.0) by constructing a standard calibration curve using serial dilutions of $\beta$-actin DNA of known concentration, from which the concentration of an unknown sample was determined. In order to analyze inter-assay variation, a least square curve fitting test was used to construct a fit. The standard error of the data points to the curve was estimated by plotting the log of the sample concentration against the number of cycles the PCR machine undertook to yield a set of fluorescent data. The standard error was estimated to be $15 \%$ on the $\log$ and was within the values described for $\beta$-actin quantification using competitive PCR.

Zymography. Lung samples $(20 \mathrm{mg} / \mathrm{ml})$ from the control and experimental animals were homogenized in $10 \mathrm{mM} 3-[(3-\mathrm{chol}-$ amidopropyl)dimethylammonio]-1-propanesulfonate (Sigma), $20 \mathrm{mM}$ HEPES (pH 7.5) plus $150 \mathrm{mM} \mathrm{NaCl}$. Electrophoresis using $7.2 \%$ polyacrylamide gels containing $2 \mathrm{mg} / \mathrm{ml}$ gelatin in non-reducing conditions $\left(4^{\circ} \mathrm{C}\right)$ was employed to determine the molecular weight distribution and general gelatinase activity of lung homogenates using methods similar to those described by Hibbs et al (13). Samples were run on duplicate gels for $\sim 1.5 \mathrm{~h}$ at $100 \mathrm{~V}$ with 10 -well minigels and a $4 \%$ stacking gel. After electrophoresis, the gels were rinsed for $30 \mathrm{~min}$ with Triton X-100 (vol/vol) to remove SDS, washed twice in $50 \mathrm{mM}$ Tris- $\mathrm{HCl}(\mathrm{pH} 7.6)$ and then incubated in $50 \mathrm{mM}$ Tris- $\mathrm{HCl}$, $5 \mathrm{mM} \mathrm{CaCl}_{2}, 1 \mu \mathrm{M} \mathrm{ZnCl}_{2}, 0.02 \% \mathrm{Brij}^{3} 3$, and $0.02 \% \mathrm{NaN}_{3}$ at $37^{\circ} \mathrm{C}$ for $24-48 \mathrm{~h}(\mathrm{pH} \mathrm{7.6)}$. After the incubation, the gels were stained with Coomassie Brilliant Blue and de-stained in methanol-acetic acid-water until clear bands of enzymatic

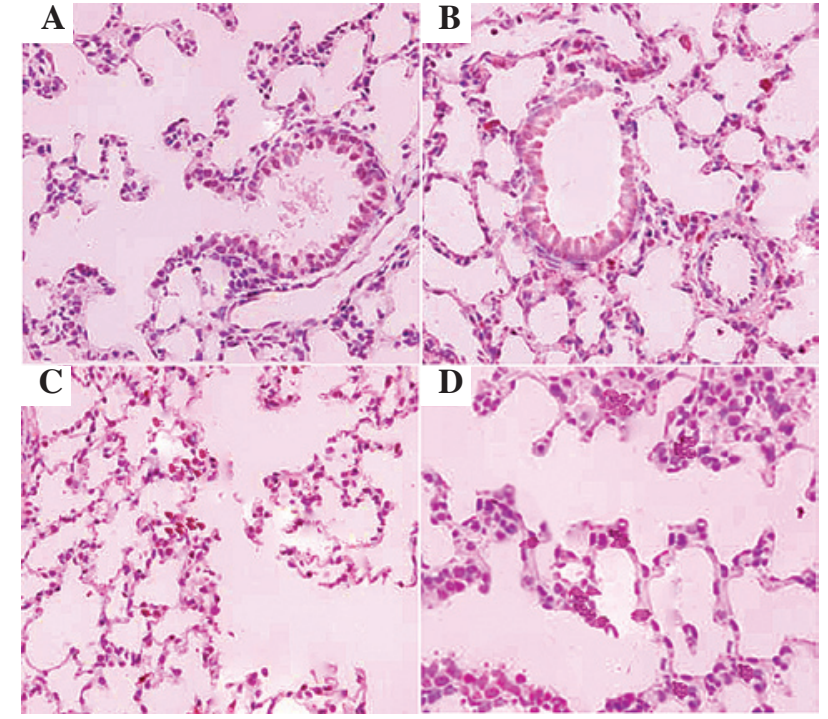

Figure 1 Light micrographs of lungs in control (A) and PQ-treated rats on days 1 (B), 3 (C) and 7 (D) after the PQ treatment (x400). Deparaffinized lung sections from animals sacrificeded at the indicated sampling intervals were prepared for hematoxylin and eosin staining. The central component of the alveolar wall is the capillary and its associated connective tissue. Large alveolar macrophages were found in the alveolar wall or free in the alveolar space.

activity were at optimal contrast from the blue staining gelatin background. Molecular weight standards were run on one lane of each gel. Gelatinolytic activities were quantified using 1D image analysis software (Eastman Kodak, Rochester, NY, USA) considering the area and intensity of lysis bands. Results were expressed as relative units (net intensity $\times 10^{-4}$ per $10 \mu \mathrm{g}$ lung protein).

Statistical analysis. SPSS 13.0 software for Windows (SPSS Inc., IL, USA) was used for statistical analysis. Data were expressed as the means \pm SD and analysed by one-way ANOVA with Tukey's post hoc test applied for paired comparisons. A difference between means was considered significant if the P-value was $<0.05$.

\section{Results}

General characteristics and histological evaluation. Rats in the PQ treatment group exhibited a constant loss of body weight (Table I) and progressive respiratory insufficiency as of day 1 after the PQ administration. Approximately $40 \%$ $(16 / 40)$ of these rats suffered acute weight loss and severe respiratory distress.

The histological appearance of the lungs is shown in Fig. 1. The control animals at all time points exhibited normal 


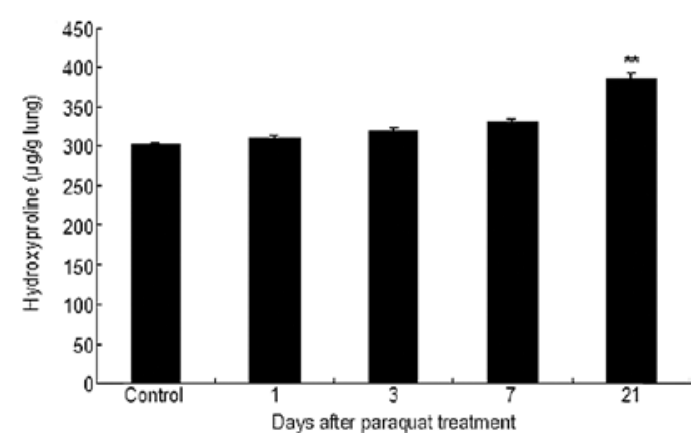

Figure 2. Hydroxyproline contents of lung tissues were significantly higher in the PQ-treated rats than the control rats on day 21. Data represent the means \pm S.D. ${ }^{*} p<0.05$ and ${ }^{* *}$ p $<0.01$ vs. the control by one-way ANOVA with Tukey's post hoc test.

A

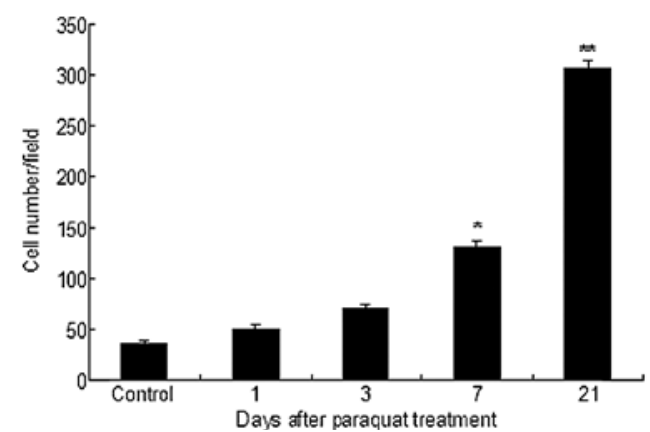

B

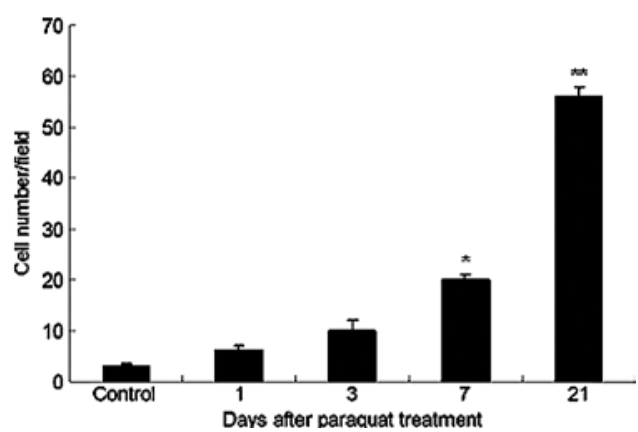

Figure 3. Immunohistochemical staining of lung tissues from PQ-treated rats and control rats. The number of alveolar macrophages (A) or T-cells (B) per high-power microscopic field were counted. Data represent the means \pm SD. ${ }^{*} \mathrm{p}<0.05$ and ${ }^{* *} \mathrm{p}<0.01$ vs. the control by one-way ANOVA with Tukey's post hoc test.

alveolar architecture (Fig. 1A). Examination of random fields under a light microscope revealed progressively disorganized lung structure, and infiltration of inflammatory cells increased in the interstitium and airspaces as the PQ-treated rats aged. Alveolar hemorrhage and capillary stasis were mostly found in rats on day 3 after the PQ treatment; foci of densely-packed interstitial fibroblasts were also found (Fig. 1C). At day 7, inflammatory cellular infiltrate was similar, but a more extensive collagen deposit was observed, as was the severe loss of parenchymal lung architecture (Fig. 1D).

Effects of $P Q$ treatment on hydroxyproline content of lung tissue. $\mathrm{PQ}$-treated rats revealed a progressive increase in lung collagen compared with the controls. As shown in Fig. 2, lung collagen quantified by hydroxyproline was increased over control levels on days 1,3 and 7 , reaching a maximum on day 21 .
A

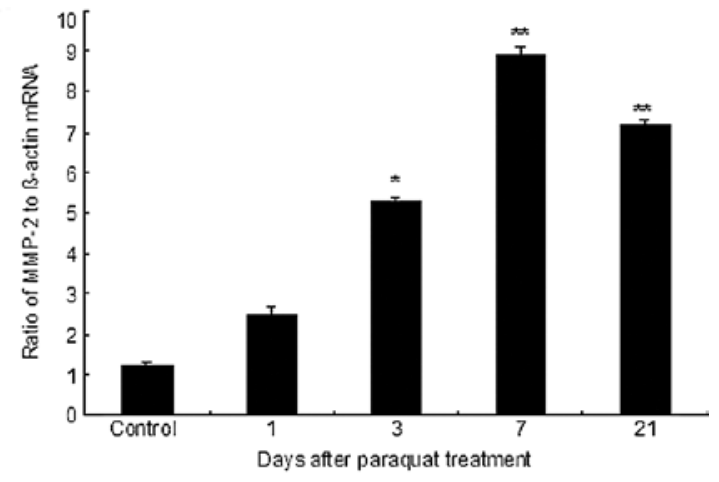

B

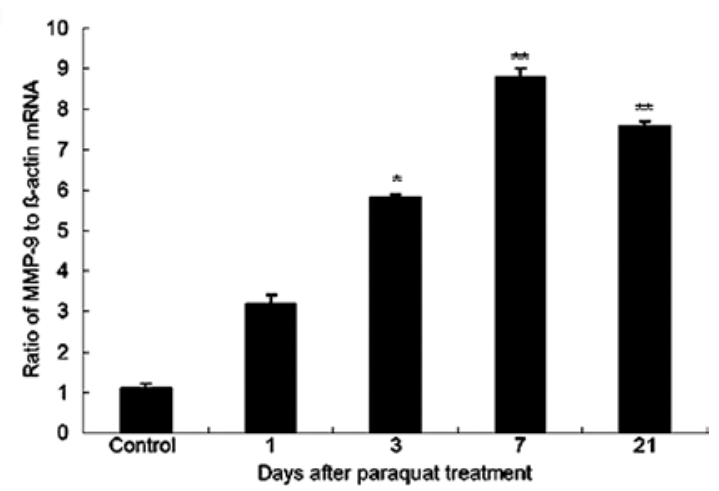

C

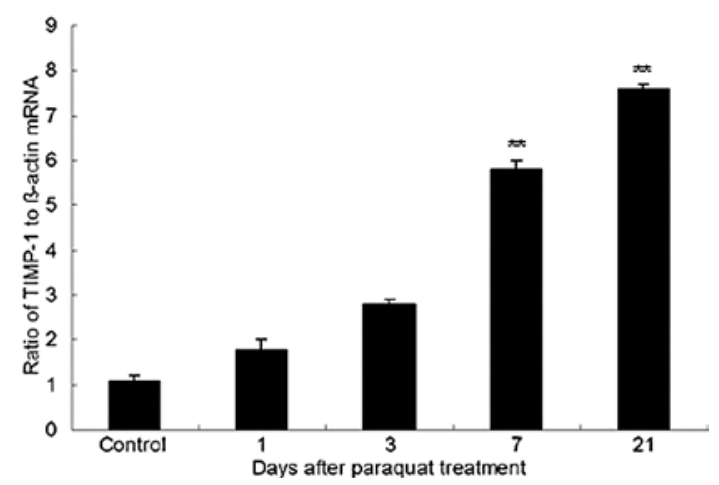

Figure 4. MMP-2 (A), MMP-9 (B) and TIMP-1 (B) gene expression in the control and PQ-treated rat lungs. Data represent the means \pm SD. " $p<0.05$ and ${ }^{* * *} \mathrm{p}<0.01$ vs. the control by one-way ANOVA with Tukey's post hoc test.

Immunohistochemical analysis of $P Q$-treated rat lungs. In order to identify the type of infiltrating cells, immunohistochemical analysis of the lung tissue from the PQ-treated rats was performed. In the lung tissue of rats without the PQ administration, several alveolar macrophages identified by staining with anti-F4/80 antibody were observed. After the PQ treatment, the number of alveolar macrophages increased significantly at day 7 after the PQ treatment, reaching a maximum on day 21 (Fig. 3A). The infiltration of T-cells into the lung tissue was also evaluated using an anti-CD3 antibody. In the lungs of the control rats, only scarce infiltration of CD3-positive T-cells was observed. PQ treatment caused T-cell recruitment in the lung. The value was increased over control levels on days 1, 3 and 7 , reaching a maximum on day 21 (Fig. 3B).

Effects of $P Q$ treatment on MMP-2, MMP-9 and TIMP-1 expression in lung tissues. Lung MMP-2 mRNA expression progressively increased after the PQ treatment, reaching a peak and statistical significance on day 7 compared to the control 
A

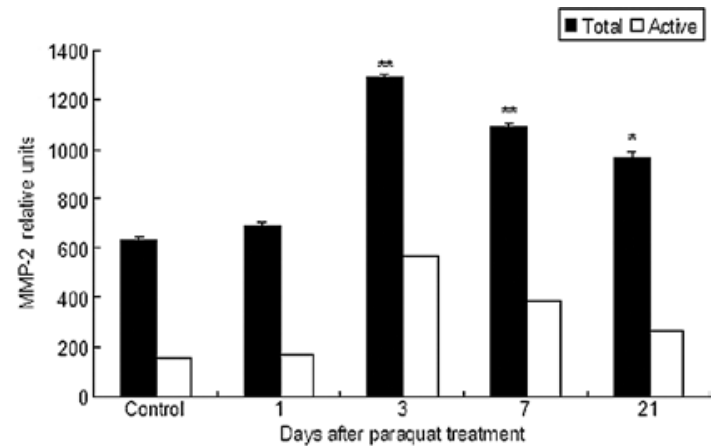

B

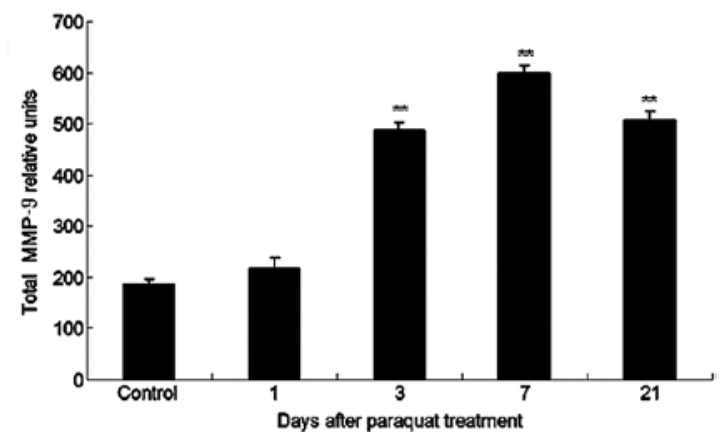

Figure 5. Lung tissue zymography. Samples containing $10 \mathrm{~g}$ of protein were mixed with an equal volume of sample buffer containing 3\% SDS Serum-free conditioned medium from human lung fibroblasts was used as the MMP-2 marker (A), and medium from phorbol 12-myristate 13-acetatestimulated U2-OS cells served as a marker of MMP-9 (B). " $p<0.05$ and *"p $<0.01$ vs. the control by one-way ANOVA with Tukey's post hoc test.

group (Fig. 4A). MMP-9 mRNA expression was comparable between the control and PQ-treated lungs on days 1 and 3, but was significantly increased by day 7 (Fig. 4B). TIMP-1 mRNA expression was significantly increased on days 7 and 21 after PQ treatment compared to the control group (Fig. 4C).

Lung tissue zymography. Compared with the control rats, PQ-treated rats revealed, from day 3 and on, an increase in pro-MMP-2 and its active form, represented by the lower molecular band of $62 \mathrm{kDa}$. A 1.5 - to 2.0 -fold increase was revealed by densitometric quantification of the surface and the intensity of the lysis bands in zymograms derived from ten rats in each group (Fig. 5A). Likewise, an increase in the band corresponding to MMP-9 was observed mainly on days 3, 7 and 21 in the PQ-treated rats (Fig. 5B). At these time points, densitometric quantification revealed a 2.5 - to 3.0 -fold increase compared to the controls.

\section{Discussion}

The major cause of death after PQ poisoning is acute respiratory distress syndrome (ARDS), which is a rather heterogenous disorder. The clinical course of this disease is divided into three phases (13): a) an early exudative phase of lung inflammation and edema; b) a proliferative phase with pneumocyte and fibroblast proliferation, and c) a final fibrotic phase with collagen deposition and pulmonary fibrosis, which is a determinant of the prognosis associated with ARDS. Previous studies with rabbits have revealed that the lung is susceptible to PQ injury following intrabronchial deposition and inhalation exposure, although it is refractory following oral or intraperitoneal administration $(14,15)$. Local instillation of PQ in the lungs of rats also produces local injury and fibrosis. Here, we prepared of a PQ-induced lung-injury rat model, with injuries similar to those observed in the lungs of PQ-poisoned patients: acute fulminated poisoning leading to death within a few days, and a more protracted form that may last for a few weeks, resulting in fatal pulmonary fibrosis. On day 21 after the PQ treatment, significant pulmonary fibrosis was evident. Collagen accumulation was indicated by trichrome staining in fibrotic interstitial tissues. Persistent inflammation and abnormal lung architecture characterized by extensive fibroblast proliferation and collagen-stained matrix deposition were observed.

In the inflammatory response after the PQ treatment, different leukocyte subsets temporally appeared within the lung (16). Previous studies demonstrated that bleomycininduced pulmonary fibrosis was independent of the accumulation of neutrophils or eosinophils $(17,18)$. There is a discrepancy in the role of T-cells in the development of pulmonary fibrosis. The depletion of T-cells attenuated bleomycin-induced pulmonary fibrosis, whereas bleomycin-challenged SCID mice lacking T-cells exhibited severe pulmonary fibrosis. By contrast, the impairment of macrophage accumulation in the lungs attenuated pulmonary fibrosis, since the alveolar macrophages secreted fibrogenic factors. In the present study, we observed an increased number of alveolar macrophages and T-cells, which indicated that PQ treatment caused alveolar macrophages and T-cell recruitment in the lung.

However, the molecular mechanisms underlying timedependent PQ-induced lung injury remain elusive. In this study, we found that increased MMP-2 and -9 mRNA expression preceded the increase in TIMP-1 mRNA expression and increased hydroxyproline content following the increase in MMP-2, MMP-9 and TIMP-1 levels in the lung tissues. These findings support the notion that an imbalance between the process of matrix degradation and their inhibition actively participates in the development of lung fibrogenesis, and that the progression of fibrosis is associated with the inhibition of matrix breakdown. In addition, MMP-2 displayed an important increase, mainly in the lung active form, as of day 3 after the PQ treatment. This finding suggests an up-regulation of membrane type 1 MMP. Results in the zymograms regarding MMP-9 also indicate an increase in MMP-9 protein. MMP-2 and -9 activity has broad substrate specificity, which includes type IV collagen, the main component of the basement membrane. Increased activity of these enzymes may contribute to the disruption of the basement membrane. In this context, it has been suggested that epithelial basement membrane integrity plays an important role in determining normal lung re-epithelialization or fibrotic response.

MMPs are zinc-dependent proteases that cleave the proteins of the extracellular matrix (ECM). They also break down the molecules that mediate cell-cell and cell-ECM interactions, and cleave and activate growth factors and growth factor receptors (19). The regulation of MMPs is controlled at several levels, including gene transcription and activation of a latent enzyme. Locally, in the extracellular space, MMPs are tightly regulated by TIMPs. In idiopathic pulmonary fibrosis, gelatinase B (MMP-9) expression is increased in cultured alveolar macrophages and in bronchoalveolar lavage fluid 
from patients (20). In bleomycin-induced pulmonary fibrosis, MMP-2 is localized in structural cells, such as regenerated alveolar epithelial cells, bronchial epithelial cells and fibroblasts (21), and may play a role in the regeneration of alveolar epithelial cells. The expression of the two gelatinases (MMP-2 and MMP-9) during the progression of fibrosis suggests that MMP-9 may be linked to inflammation-induced tissue remodeling, while MMP-2 may be associated with impaired tissue remodeling, leading to pathological collagen deposition and interstitial fibrosis. In line with this observation, the overexpression of TIMPs has been observed, leading to an imbalance between MMPs and their inhibitors and resulting in a microenvironment unfavorable to collagenolytic activity (22). TIMP-1, -2 and -3 are produced by different cells and play distinct roles in MMP activity regulation. TIMP-2 is mainly localized in regenerated epithelial cells and fibroblasts (23). TIMP-1 is primarily expressed on alveolar macrophages and epithelial cells, and TIMP-2 and -3 genes are constitutively expressed, whereas TIMP-1 gene expression is up-regulated during fibrosis (24). TIMP-1 expression is correlated with the stages of disease progression, may prevent MMP-induced extracellular matrix degradation and, as a consequence, may participate in the accumulation of extracellular matrix $(25,26)$.

In conclusion, unbalanced MMP/TIMP-1 expression and excessive gelatinolytic activity contribute to PQ-induced pulmonary fibrosis. Their precise role must be studied in depth as they may represent relevant therapeutic targets for PQ poisoning-induced pulmonary fibrosis.

\section{References}

1. Brian RC, Homer RE, Stubbs J and Jones RL: A new herbicide, 1: 1-ethylene-2, 21-dipyridylium dibromide. Nature 181: 446, 1958.

2. Clark DG, McElligott TE and Weston HE: The toxicity of paraquat. Br J Ind Med 23: 126-132, 1966.

3. Tomita M, Okuyama T, Katsuyama H, Hidaka K, Otsuki T and Ishikawa T: Gene expression in rat lungs during early response to paraquat-induced oxidative stress. Int J Mol Med 17: 37-44, 2006.

4. Shou Y, Hirano T, Gong Y, et al: Influence of angiogenetic factors and matrix metalloproteinases upon tumour progression in non-small cell lung cancer. Br J Cancer 85: 1706-1712, 2001.

5. Sounni NE and Noel A: Membrane type-matrix metalloproteinases and tumor progression. Biochimie 87: 329-342, 2005

6. Ylisirnio S, Hoyhtya M and Turpeenniemi-Hujanen T: Serum matrix metalloproteinases-2, -9 and tissue inhibitors of metalloproteinases-1, -2 in lung cancer - TIMP-1 as a prognostic marker. Anticancer Res 20: 1311-1316, 2000.

7. Brew K, Dinakarpandian D and Nagase H: Tissue inhibitors of metalloproteinases: evolution, structure and function. Biochim Biophys Acta Prot Struct Mol Enzymol 1477: 267-283, 2000.

8. Cataldo D, Munaut C, Noel A, et al: MMP-2-and MMP-9-linked gelatinolytic activity in the sputum from patients with asthma and chronic obstructive pulmonary disease. Int Arch Allergy Immunol 123: 259-267, 2000.
9. Cataldo D, Munaut C, Noel A, et al: Matrix metalloproteinases and TIMP-1 production by peripheral blood granulocytes from COPD patients and asthmatics. Allergy 56: 145-151, 2001.

10. Cataldo D, Gueders M, Noel A, Foidart JM, Bartsch P and Louis R: Matrix metalloproteinases (MMPs) and tissue inhibitors of matrix metalloproteinases (TIMPs) mRNA transcripts in the bronchial secretions of asthmatics and COPD. Lab Invest 84: 418-424, 2002

11. Cataldo DD, Tournoy KG, Vermaelen K, et al: Matrix metalloproteinase-9 deficiency impairs cellular infiltration and bronchial hyperresponsiveness during allergen-induced airway inflammation. Am J Pathol 161: 491-498, 2002.

12. Woessner JF: The determination of hydroxyproline in tissue and protein samples containing small proportions of this amino acid. Arch Biochem Biophys 93: 440-447, 1961.

13. Tomita M, Okuyama T, Katsuyama H, et al: Mouse model of paraquat-poisoned lungs and its gene expression profile. Toxicology 231: 200-209, 2007.

14. Tomita M, Okuyama T, Hidaka K, Ishikawa T, Adachi J and Nohno T: Early differential gene expression of rat lung after exposure to paraquat. Free Radic Res 38: 821-829, 2004.

15. Yeh ST, Guo HR, Su YS, et al: Protective effects of Nacetylcysteine treatment post-acute paraquat intoxication in rats and in human lung epithelial cells. Toxicology 76: 530-537, 2006.

16. Tomita M, Nohno T, Okuyama T, Nishimatsu S and Adachi J: Paraquat-induced gene expression in rat lung tissues using a differential display reverse transcription-polymerase chain reaction. Arch Toxicol 76: 530-537, 2002.

17. Hagimoto N, Kuwano K, Nomoto Y, Kunitake R and Hara N: Apoptosis and expression of Fas/Fas ligand mRNA in bleomycininduced pulmonary fibrosis in mice. Am J Respir Cell Mol Biol 16: 91-101, 1997.

18. Haston CK, Tomko TG, Godin N, Kerckhoff L and Hallett MT: Murine candidate bleomycin induced pulmonary fibrosis susceptibility genes identified by gene expression and sequence analysis of linkage regions. Med Genet 42: 464-473, 2005.

19. Piotrowski WJ, Nawrocka-Kunecka A, Antczak A, et al: Metalloproteinases MMP-9, MMP-2 and their tissue inhibitors TIMP-1, TIMP-2 in peripheral transbronchial lung biopsies of patients with sarcoidosis. Pol Arch Med Wewn 119: 628-635, 2009.

20. Gaggar A, Li Y, Weathington N, et al: Matrix metalloprotease-9 dysregulation in lower airway secretions of cystic fibrosis patients. Am J Physiol Lung Cell Mol Physiol 293: 96-104, 2007.

21. Manoury B, Caulet-Maugendre S, Guénon I, Lagente V and Boichot E: TIMP-1 is a key factor of fibrogenic response to bleomycin in mouse lung. Int J Immunopathol Pharmacol 19: 471-487, 2006.

22. García-Alvarez J, Ramirez R, Sampieri CL, et al: Membrane type-matrix metalloproteinases in idiopathic pulmonary fibrosis. Sarcoidosis Vasc Diffuse Lung Dis 23: 13-21, 2006.

23. Han $Z$ and Zhong JX: Expression of matrix metalloproteinases MMP-9 within the airways in asthma. Resp Med 97: 563-567, 2003.

24. Handsley MM and Edwards DR: Metalloproteinases and their inhibitors in tumor angiogenesis. Int $\mathrm{J}$ Cancer 115: 849-860, 2005.

25. Jumper C, Cobos E and Lox C: Determination of the serum matrix metalloproteinase-9 (MMP-9) and tissue inhibitor of matrix metalloproteinase-1 (TIMP-1) in patients with either advanced small-cell lung cancer or non-small-cell lung cancer prior to treatment. Respir Med 98: 173-177, 2004.

26. Ohbayashi H: Matrix metalloproteinases in lung diseases. Curr Protein Pept Sci 3: 409-421, 2002. 